\title{
Simulation of Multiphase Fluid Motion in Pore-scale Fractures
}

\author{
M.B. Liv ${ }^{1, *}$ and J.Z. Chang ${ }^{2}$ \\ ${ }^{1}$ Institute of Mechanics, Chinese Academy of Science, Beijing 100190, China \\ ${ }^{2}$ School of Mechatronic Engineering, North University of China, Taiyuan, Shanxi 030051, China
}

\section{Introduction}

Small scale environmental and geophysical flows are very important, but are usually difficult to simulate because of the associated multiple fluid phases and multiple physics, as well as the existence of complex geometries and arbitrarily moving interfaces. For example, fluid motion in the vadose zone is very critical for groundwater recharge, fluid motion and contaminant transport. Flow through fractures and fractured porous media can lead to exceptionally rapid movement of liquids and associated contaminants. ${ }^{[1,2]}$ The physics of fluid flows in unsaturated fractures and porous media is still poorly understood due to the complexity of multiple phase flow dynamics. Experimental studies of fluid flow in fractures and fractured porous media are limited, and in computer simulations it is usually difficult to take into account the fracture surface properties and microscopic roughness. A broadly applicable model must be able to simulate a variety of phenomena including film flow with free surfaces, stable rivulets, snapping rivulets, fluid fragmentation and coalescence (including coalescence/fragmentation cascades), droplet migration and the formation of isolated single-phase islands trapped due to aperture variability.

Realistic models for multiphase fluid flows in fracture and fractured porous media must be able to handle moving interfaces, large density ratios (e.g., $\approx 1000: 1$ for water and air), and large viscosity ratios (e.g., $\approx 100: 1$ for water and air). These requirements combined with the complex geometries of natural fractures present severe challenges to mechanistic models. Grid based numerical methods such as finite difference methods, finite volume methods and Eulerian finite element methods require special algorithms to treat and track the interface between different phases. However, continuum grid based numerical models usually do not take account of the detailed void and obstacle geometries, fluid-fluid interface dynamics within pores and complex fluid-fluid-solid contact line dynamics. They rely on constitutive equations that describe the coarse-grained behaviour and can, at least in principle, be derived from the results of pore scale simulations or experiments. Therefore, small-scale simulations with mechanistic models are needed to develop a better understanding of the temporal and spatial dynamics of multiphase flow through pore-scale structures such as fractures and fractured porous media. Pore-scale flows have been studied extensively using grid based methods including finite difference method, ${ }^{[3]}$ finite volume method, ${ }^{[4]}$ and finite element method, ${ }^{[5]}$ However, due to the difficulties associated with geometrically complex boundaries, fluidfluid-solid contact line dynamics, and fluid-fluid interface dynamics, it is difficult to apply conventional grid based multiphase simulation methods coupled with interface tracking algorithms to pore-scale multiphase flow modelling.

Dissipative particle dynamics is a meso-scale particle method. Though it may be less computationally efficient than the grid-based methods, it is advantageous in simulating pore-scale multiphase flow modelling in fractures. DPD is a Lagrangian method, and conserves mass exactly. In DPD method, there is no explicit interface tracking - the motion of the fluid is

\footnotetext{
*Corresponding author. E-mail: liumoubin@imech.ac.cn
} 
represented by the motion of the particles, and fluid surfaces or fluid-fluid interfaces move with the particles. In this paper we will demonstrate the application of the DPD in simulating multiphase fluid flow in fractures with a number of numerical examples.

\section{Basic Concept of Dissipative Particle Dynamics}

Dissipative particle dynamics ${ }^{[6,7]}$ is a relatively new mesoscale technique that can be used to simulate the behaviour of complex fluids. In DPD simulations, a complex system can be simulated using a set of interacting particles. A particles represent clusters of molecules that interact via conservative (non-dissipative), dissipative and fluctuating forces. Because the effective interactions between clusters of molecules are much softer than the interactions between individual molecules, much longer time steps can be taken relative to molecular dynamics (MD) simulations. A longer time steps combined with a larger particle size makes DPD much more practical to simulate hydrodynamics than MD. DPD is particularly promising for the simulation of complex liquids, such as polymer suspensions, liquids with interfaces, colloids and gels. Because of the symmetry of the interactions between the particles, DPD rigorously conserves the total momentum of the system, and because the particle-particle interactions depend only on relative positions and velocities, the resulting model fluids are Galilean invariant. Mass is conserved because the same mass is associated with each of the particles, and the number of particles does not change.

It is convenient to assume that all of the particles have equal masses, and use the mass of the particles as the unit of mass. Newton's second law governs the motion of each particle. The time evolution for a certain particle, $i$, is given by the following equation of motion

$$
\frac{d r_{i}}{d t}=v_{i}, \frac{d v_{i}}{d t}=f_{i}=f_{i}^{i n t}+f_{i}^{e x t},
$$

where $r_{i}$ and $v_{i}$ are the position and velocity vectors of particle $i, f_{i}^{\text {ext }}$ is the external force including the effects of gravity, and $f_{i}^{\text {int }}$ is the inter-particle force acting on particle $i$. The particle-particle interaction is usually assumed to be pairwise additive and consist of three parts: a conservative (non dissipative) force, $F_{i j}^{C}$; a dissipative force, $F_{i j}^{D}$; and a random force, $F_{i j}^{R}$, i.e. $f_{i}^{\mathrm{int}}=\sum_{j \neq i} F_{i j}=\sum_{j \neq i} F_{i j}^{C}+F_{i j}^{D}+F_{i j}^{R} \cdot F_{i j}$ is the inter-particle interaction force exerted on particle $i$ by particle $j$, which is equal to $F_{j i}$ in magnitude and opposite in direction. This symmetry of the interactions ensures that momentum is rigorously conserved. The pairwise particle interactions have a finite cutoff distance, $r_{c}$, which is usually taken as the unit of length in DPD models.

The dissipative force $F_{i j}^{D}$ represents the effects of viscosity, and is given by $F_{i j}^{D}=-\gamma w^{D}\left(r_{i j}\right)$ $\left(\hat{r}_{i j} g v_{i j}\right) \hat{r}_{i j}$, where $\gamma$ is a coefficient, $r_{i j}=r_{i}-r_{j}, r=r_{i j}=\left|r_{i j}\right|, \hat{r}_{i j}=r_{i j} / r_{i j}, v_{i j}=v_{i}-v_{j}$ and $w^{D}\left(r_{i j}\right)$ is the dissipation weight function. The random force $\mathbf{F}_{i j}^{R}$ represents the effects of thermal fluctuations, and is given by $\mathbf{F}_{i j}^{R}=\sigma w^{R}\left(r_{i j}\right) \xi_{i j} \hat{r}_{i j}$, where $\sigma$ is a coefficient, $w^{R}\left(r_{i j}\right)$ is the fluctuation weight function, and $\xi_{i j}$ is a random variable. The fluctuation-dissipation relationship ${ }^{[8]}$ requires $w^{D}(r)=\left[w^{R}(r)\right]^{2}$ and $\gamma=\frac{\sigma^{2}}{2 k_{B} T}$, where $k_{B}$ is the Boltzmann constant and $T$ is the temperature. One straightforward choice for the dissipative and random weight functions is $w^{D}(r)=\left[w^{R}(r)\right]^{2}=(1-r)^{2}, r<1$.

The conservative force, $F_{i j}^{C}$, is a "soft" interaction acting along the line of particle centres, and has the form $F_{i j}^{C}=a_{i j} w^{C}(r) \hat{r}_{i j}$, where $a_{i j}$ is the magnitude of the repulsive interaction strength between particles $i$ and $j$. For particles from different media, the strength coefficient 
can be different. $w^{C}\left(r_{i j}\right)$ is the weight function for the conservative force. In previous DPD implementations, a conservative force weighting function in a simple form of $w^{C}(r)=1-r$ has been used. Because the fluid generated by DPD simulations with this purely repulsive conservative force is a gas, it cannot be used to simulate the flow of liquids with free surfaces, the behaviour of bubbly liquids, droplet dynamics and other important multiphase fluid flow processes. Including a long-range attractive component in $w^{C}(r)$ is necessary for such applications.

\section{Modification of the Interaction Potentianl Function}

We constructed a new particle-particle interaction potential $U(r)$ by combining the commonly used SPH cubic spline smoothing functions $W\left(r, r_{c}\right)$ with different interaction strengths $A$ and $B$, and different cutoff distances $r_{c 1}$ and $r_{c 2}$, multiplied by an interaction strength coefficient $a$

$$
U(r)=a\left(A W\left(r, r_{c 1}\right)-B W\left(r, r_{c 2}\right)\right)
$$

The DPD conservative particle-particle interaction forces are thus given by $F_{i j}^{C}=\frac{-d U(r)}{d r} \hat{r}_{i j}$. The constructed interaction potential function $U(r)$ consists of short-range repulsive and large range attractive interactions (when $A>B$, and $r_{c 1}<r_{c 2}$ ) and allows the behavior of gases, liquids, solids and multiphase systems to be simulated. A certain set of parameters $A, B, r_{c 1}$ and $r_{c 2}$ in equation (2) determines the shape of the particle-particle interaction potential which describes the property of the corresponding fluid. The magnitude of the conservative force weight function and the location of the transition point from repulsion to attraction should be easily adjustable to allow the behavior of different fluids to be simulated. Figure 1 shows the conventional DPD potential function, $U(r)=0.5-\left(r-0.5 r^{2}\right)$, the cubic spline potential functions, $U(r)=W(r, 1.0)$, and two particle-particle interaction potential functions resulting from the cubic spline, $U(r)=2 W(r, 0.8)-W(r, 1.0)$ and $U(r)=2 W(r, 0.8)-0.9 W(r, 1.0)$.

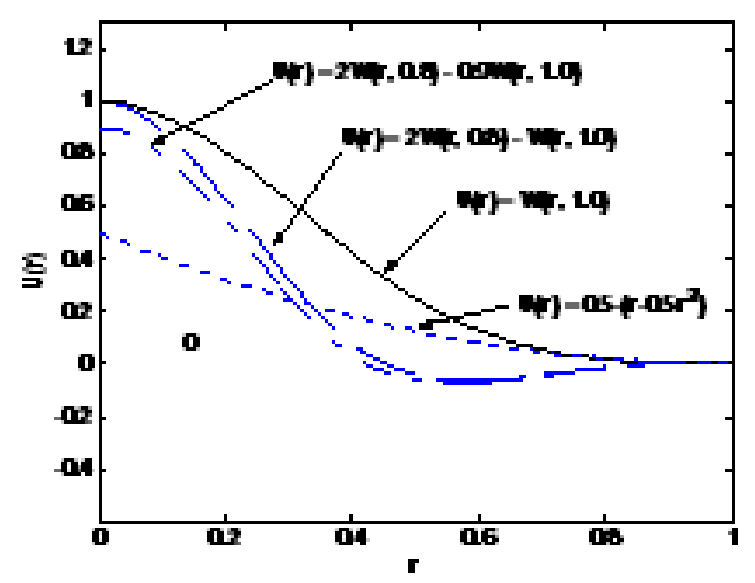

Figure 1. The conventional DPD potential function, $U(r)=0.5-\left(r-0.5 r^{2}\right)$, the cubic spline potential functions, $U(r)=W(r, 1.0)$, and two potential functions resulting from the cubic spline, $U(r)=2 W(r, 0.8)-W(r, 1.0)$ and $U(r)=2 W(r, 0.8)-0.9 W(r, 1.0)$. 
In DPD simulations, the effects of solid walls are usually be simulated by using fixed particles to represent the solid matrix near the solid-fluid interface. In our implementation, the entire computational domain is covered by a grid and grid cells are labeled " 0 " for fluid filled regions and " 1 " for solid filled regions. DPD particles are injected into the computational domain randomly until a pre-defined particle number density is reached, and the system is then run to equilibrium using a DPD simulation. The particles within the solid cells (marked as " 1 ") are then 'frozen' to represent the solid matrix. In order to reduce computational effort only the frozen particles that are within 1 DPD unit $\left(\right.$ or $\left.r_{c}\right)$ from the solid-fluid interface are chosen as boundary DPD particles. The random distribution of the frozen wall particles describes the solid surface roughness in a natural way. A reflective boundary was used in addition to the interactions between fluid and wall particles. The implementation of no-slip boundary conditions with randomly distributed frozen wall particles was found to be very flexible, especially for problems with complex geometries such as porous media and fracture geometries. ${ }^{[9]}$

\section{Multiphase Flow in a Fractured Junction With Fractal Surfaces}

The geometry of the fracture junction is shown in Figure 2, in which the fracture walls were represented by self-affine fractal surfaces characterized by a Hurst exponent. ${ }^{[10]}$ The size of the computational domain is $128 \times 3 \times 128$ in the $x, y$ and $z$ direction. The fracture walls were represented by 7116 frozen wall particles. The coefficients used in the DPD model were $\sigma=3.0$ and $k_{B} T=1.0(\gamma=4.5)$. The interaction strength between the fluid particles was $a_{f}=18.75$, and $a_{w}$, the strength of the interactions between the fluid and wall particles, can be changed to mimic different wetting behaviors. A modified velocity-Verlet time integration algorithm was used for time integration. ${ }^{[7]}$ The parameters for the SPH potential and weight functions were $A=2.0, r_{c 1}=0.8, B=1.0$ and $r_{c 2}=1.0$. The particle-particle interaction potentials were given by $U(r)=a_{f}\left(2 W_{1}(r, 0.8)-W_{2}(r, 1.0)\right)$ for fluid-fluid particle interactions and $U(r)=a_{w}\left(2 W_{1}(r, 0.8)-W_{2}(r, 1.0)\right)$ for fluid-wall particle interactions.

Figure 2 shows the particle distribution of an injection flow into the fracture junction from the top fracture aperture, with an injection rate of 200 particles per 100 steps, an interaction ratio $a_{w} / a_{f}$ of 5 , and gravitational forces, $g_{x}=-0.02$, and $g_{z}=-0.02$ along negative $x$, and $z$ direction. A few particles evaporated from the bulk fluid and the flow was a twophase flow with co-existing liquid-gas phases. The particles near the fracture walls moved into the aperture faster than those far from the walls, and the fluid exhibited a strong wetting behaviour with a small contact angle. In contrast to grid-based methods in which the contact angle is imposed on the fluid, the contact angle in DPD is estimated from the position of the wall and liquid particles. The strong wettability of the fluid leaded to a continuous film flow

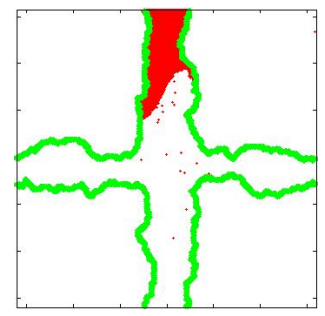

(a)



(b)

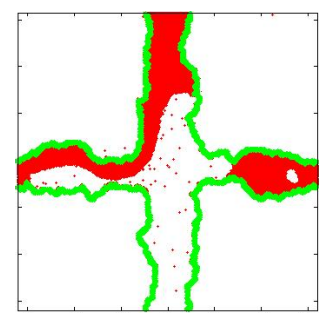

(c)

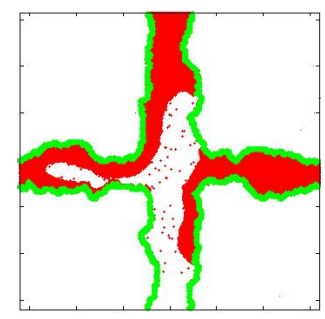

(d)

Figure 2. Sequential images of an injection into the fracture junction at (a) 1000, (b) 20000, (c) 30000 and (d) 40000 steps obtained using DPD method. 


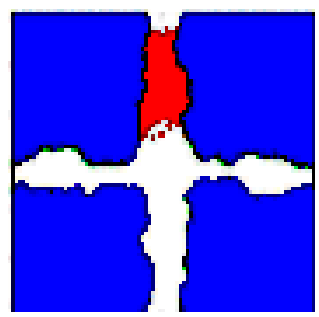

(a)

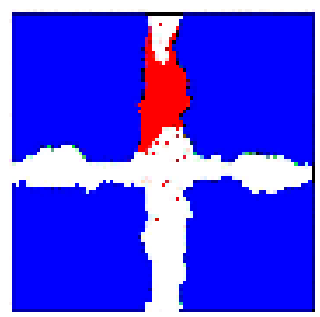

(b)

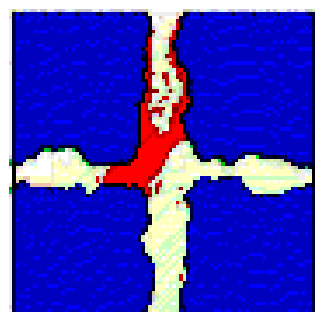

(c)

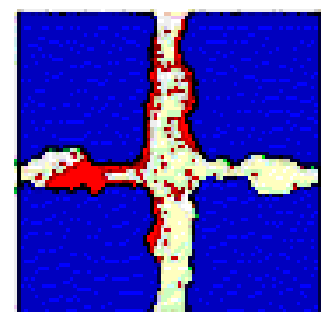

(d)

Figure 3. Sequential images of flow of a liquid droplet through the fracture junction at (a) 300, (b) 1100 , (c) 2500, and (d) 4300 steps obtained using DPD method.

formed along the fracture wall surface (Figure 2b, c, and d). A gas bubble was entrapped in the bulk liquid due to the wetting effects and fracture aperture variation (Figure 2c).

Figure 3 shows the sequential images of flow of a liquid droplet through the fracture junction with an interaction ratio $a_{w} / a_{f}$ of 3 , and gravitational forces, $g_{x}=-0.05$, and $g_{z}=$ -0.05 , along negative $x$, and $z$ direction. The gravitational force was large enough to offset the capillary and viscous forces. Therefore, the liquid drop broke at the fracture junction, and entered the left horizontal and lower vertical apertures. Some liquid was disconnected from the bulk fluid, and formed thin films along the fracture walls. The contact angles of the wetting fluid varied at the upstream and downstream of the bulk liquid, and demonstrated as advancing and receding contact angles. The advancing and receding contact angles varied with position and time. Further investigation revealed that the contact angle was closely related to $a_{f}, a_{w}$ and $g$, whereas $a_{f}, a_{w}$ and $g$, characterized the dynamic balance of viscous, capillary, and gravitational forces. This velocity dependent contact angle behavior was also observed in real systems. ${ }^{[11]}$

\section{Multiphase Flow Through a Porous Media Overlying a Fracture With Fractal Surfaces}

The geometry of this case is shown in Figure 4, which is a heterogeneous granular porous overlying a fracture with self-affine fractal surfaces. The size of the computational domain is $128 \times 2 \times 128$ in $x, y$ and $z$ direction. The fracture walls were represented by 9734 frozen wall particles. In $x, y$ and $z$ direction, periodic boundary was applied, where on fracture surfaces, no-slip boundary was imposed. The coefficients used in the DPD model were $\sigma=$ 3.0 and $k_{B} T=1.0(\gamma=4.5)$. The interaction strength between the fluid particles was $a_{f}=$ 18.75 , and the interaction ratio is $a_{w} / a_{f}=0.5$. The parameters for the SPH potential and weight functions were $A=2.0, r_{c 1}=0.8, B=1.0$ and $r_{c 2}=1.0$. Figure 4 shows the injection of liquid into the porous media with an injection rate of 100 particles per 100 steps, and a downward vertical gravitational force, $g$, of 0.05 . Since the interaction between fluid particles, $a_{f}$, is larger than the interaction between fluid and wall particles, $a_{w}$, the liquid exhibits a non-wetting behaviour with a contact angle larger than $\pi / 2$. The contact angle varied with position and time due to the dynamic balance between viscous, capillary, and gravitational forces. The injected fluid particles moved downward, through the porous media, as the density of the injected particles and the concomitant pressure increased, and then reached the fracture aperture. The gravitational force was large enough for some liquid to break from the bulk liquid and form some small liquid drops. 




(a)

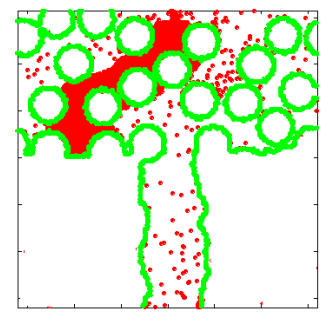

(b)



(c)

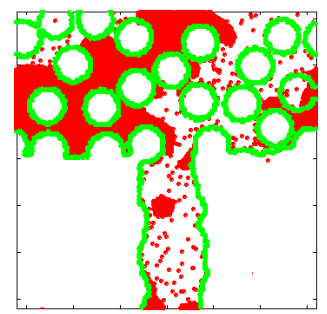

(d)

Figure 4. Sequential images of an injection into a porous media overlying a fracture at (a) 1000, (b) 20000, (c) 40000, and (d) 55000 steps obtained using DPD method.

\section{Multiphase Flow in a Fracture Network}

A numerical study using a volume of fluid (VOF) method was presented by Huang and his coworkers to investigate the unsaturated multiphase flow through a fracture network, together with a flow experiment based on the same fracture network fabricated using polymethylmethacrylate. ${ }^{[12]}$ Here a DPD simulation was also conducted for the same fracture network geometry. The size of the computational domain is $100 \times 3 \times 103$ in DPD unit in $x, y$ and $z$ direction. The fracture walls were represented by 13844 frozen wall particles. In $x, y$ and $z$ direction, periodic boundary was applied, where on fracture surface, no-slip boundary was imposed. The coefficients used in the DPD model were $\sigma=3.0$ and $k_{B} T=1.0(\gamma=4.5)$. The interaction strength between the fluid particles was $a_{f}=18.75$, and the interaction ratio is $a_{w} / a_{f}=5$. The parameters for the SPH potential and weight functions were $A=2.0, r_{c 1}=$ $0.8, B=1.0$ and $r_{c 2}=1.0$. In the simulation, the gravitational force was taken 0.3 in DPD unit. In the flow experiment, the apparatus was tilted $2.5^{\circ}$ in the plane of the photograph. This corresponds to a diagonal gravitational force with a component along both the leftward horizontal and downward vertical directions. The injection was conducted at two DPD cells along the left fracture wall of the top entrance. This corresponds to the injection of water into the top entrance of the channel network using a syringe pump positioned next to the left top fracture aperture.

Figure 5 shows the comparisons of the injection flow into the channel network at an intermediate stage obtained from DPD model (Figure 5a), VOF model (Figure 5b), and (3) exper-

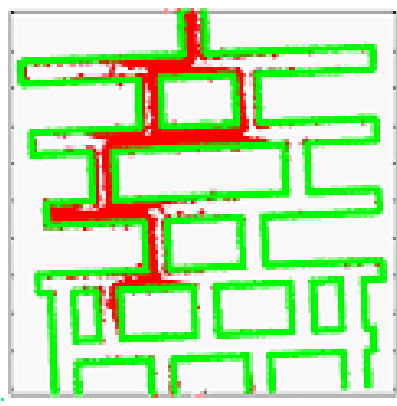

(a)

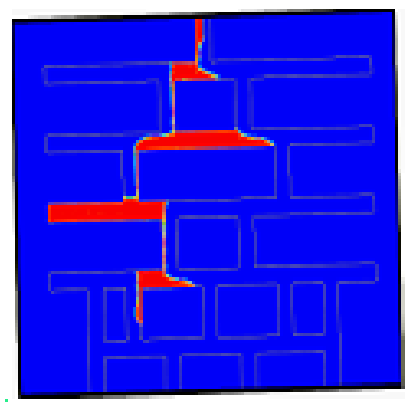

(b)

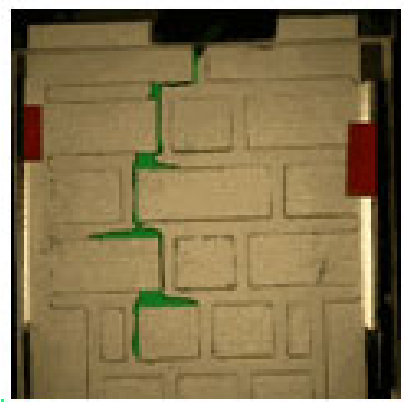

(c)

Figure 5. Comparisons of the injection flow into the channel network at an intermediate stage from DPD model (a), VOF model (b), and (3) experimental observation (5c). 
imental observation (Figure 5c). It is clear that the DPD simulation result agrees qualitatively with the experimental observation and VOF result provided in. ${ }^{[12]}$ There are some discrepancies if precisely examining the figures from the DPD simulation, VOF simulation and experiment. This is understandable since even for very simple fracture geometries, the multiphase flow can exhibits very complex spatial and temporal behaviours. Small perturbations in the simulation and experiment can give rise to quite different flow modes. The VOF simulation assumed constant fracture aperture with smooth fracture surface, and the advancing and receding contact angles were prescribed to be constant. The fracture walls in the experiment are more or less rough rather than smooth in micro-scale, which can affect the wetting behaviour and flow modes of the fluid. The DPD simulation used randomly distributed frozen wall particles, whose positions determine the roughness of the fracture wall surfaces, and affect the advancing and receding contact angles.

\section{Conclusions}

This paper presented the simulations of multiphase flow in complex pore-scale fracture geometries using a dissipative particle dynamics method. This dissipative particle dynamics method employed conservative particle-particle interactions that combine short-range repulsive and long-range attractive interactions to simulate gases, liquids, solids and multiphase systems, depending on the average particle density, the temperature and the details of the particle-particle interactions. The interaction strength between the fluid particles, and between the fluid and wall particles are closely related to the wetting behaviour and the contact angles.

The simulations revealed that multiphase flow in pore-scale fracture geometries is complicated due to the interplay of viscous, capillary and gravitational forces, fracture geometry, and the inflow conditions. Different flow modes can coexist in a complex fracture geometry system, including continuous or discontinuous film flow, entrapment of one phase in another one, stationary or moving droplets. Small perturbations of the flow regime or fracture surface can give rise to quite different flow modes. The advancing and receding contact angles can vary spatially and temporally, depending on the dynamic balance of viscous, capillary and gravitational forces.

\section{References}

1. Scanlon, B. R., Tyler S. W. and Wierenga P. J., "Hydrologic issues in arid, unsaturated systems and implications for contaminant transport", Reviews of Geophysics, 35, 4, 1997, pp. 461.

2. Nativ, R., Adar E., Dahan O. and Geyh M., "Water recharge and solute transport through the vadose zone of fractured chalk under desert conditions", Water Resources Research, 31, 2, 1995, pp. 253.

3. Anderson, J. D., Computational fluid dynamics: The basics with applications, McGraw Hill, New York, 2002.

4. Chung, T. J., Computational fluid dynamics, Cambridge University Press, 2002.

5. Zienkiewicz, O. C. and Taylor R. L., The finite element method, Butterworth-Heinemann, 2000.

6. Hoogerbrugge, P. J. and Koelman J., "Simulating microscopic hydrodynamic phenomena with dissipative particle dynamics”, Europhysics Letters, 19, 1992, pp. 155.

7. Groot, R. D., "Dissipative particle dynamics: Bridging the gap between atomistic and mesoscopic simulation", Journal Of Chemical Physics, 107, 11, 1997, pp. 4423.

8. Espanol, P. and Warren P., "Statistical mechanics of dissipative particle dynamics", Europhysics Letters, 30, 4, 1995, pp. 191.

9. Liu, M. B., Meakin P. and Huang H., "Dissipative particle dynamics simulation of pore-scale flow”, Water Resources Research, 43, 2007. 
October 31, 2009 15:30 RPS ICADD09-126

Analysis of Discontinuous Deformation: New Developments and Applications

10. Meakin, P., Fractals, scaling and growth far from equilibrium, Cambridge university press, 1998.

11. Cox, R. G., "Inertial and viscous effects on dynamic contact angles", Journal of Fluid Mechanics, 357, 1998, pp. 249.

12. Huang, H., Meakin P., Liu M. B. and McCreery G. E., "Modeling of multiphase fluid motion in fracture intersections and fracture networks", Geophysical Research Letters, 32, 2005. 